\title{
Lifestyle Patterns Begin in Early Childhood, Persist and Are Socioeconomically Patterned, Confirming the Importance of Early Life Interventions
}

\author{
Sandrine Lioret ${ }^{1, *}$, Karen J. Campbell ${ }^{2}$, Sarah A. McNaughton ${ }^{2}$, Adrian J. Cameron ${ }^{3}$, \\ Jo Salmon ${ }^{2}$, Gavin Abbott ${ }^{2}$ (D) and Kylie D. Hesketh ${ }^{2}$ \\ 1 Research Center in Epidemiology and Biostatistics (CRESS), Université de Paris, INSERM, INRA, \\ 75004 Paris, France \\ 2 Institute for Physical Activity and Nutrition (IPAN), School of Exercise and Nutrition Sciences, \\ Deakin University, Geelong, VIC 3220, Australia; karen.campbell@deakin.edu.au (K.J.C.); \\ sarah.mcnaughton@deakin.edu.au (S.A.M.); jo.salmon@deakin.edu.au (J.S.); \\ gavin.abbott@deakin.edu.au (G.A.); kylie.hesketh@deakin.edu.au (K.D.H.) \\ 3 Global Obesity Centre (GLOBE), Institute for Health Transformation, School of Health and Social \\ Development, Deakin University, Geelong, VIC 3220, Australia; adrian.cameron@deakin.edu.au \\ * Correspondence: sandrine.lioret@inserm.fr; Tel.: +33-1-45-59-51-78
}

Received: 27 January 2020; Accepted: 5 March 2020; Published: 9 March 2020

\begin{abstract}
Traditional approaches to understanding the behavioural determinants of adiposity have considered diet, physical activity and sedentary behaviour in isolation. Although integrative approaches have identified a variety of lifestyle patterns in children at preschool-age or older, along with some variability by socio-economic positions, this has rarely been examined in younger cohorts. We aimed to identify lifestyle patterns at 1.5, 3.5 and 5 years, including dietary intake, outdoor time and television viewing time, to assess associations with maternal education (as a proxy for socio-economic position), and to investigate their persistence between toddlerhood and preschool age. Participants were 417 and 293 children aged 1.5 y from the Melbourne Infant Feeding Activity and Nutrition Trial (InFANT) and InFANT Extend Programs, respectively. Data were collected using questionnaires at child ages 1.5, 3.5 and $5 \mathrm{y}$ (InFANT); and 1.5 and $3.5 \mathrm{y}$ (InFANT Extend). Principal component analysis was undertaken at each time point on the separate and pooled datasets. Associations between the lifestyle patterns scores and maternal education were assessed with multivariable regression analysis. Two lifestyle patterns ("Discretionary consumption and TV" and "Fruit, vegetables and outdoor") were identified as early as $1.5 \mathrm{y}$. They remained consistent across ages and were evident in both datasets. These patterns were inversely and positively associated with maternal education, respectively. Such early clustering of obesity related energy balance behaviours and tracking during early childhood suggests there may be shared antecedents common to the individual behaviours that could be targeted for intervention. Our findings provide support for interventions targeting multiple behaviours and tailored to the level of family socio-economic disadvantage.
\end{abstract}

Keywords: diet; physical activity; sedentary behaviour; energy balance-related behaviours; lifestyle patterns; early childhood; tracking

\section{Introduction}

Childhood overweight and obesity is common from as early as two years of age and increases the probability of persistent unhealthy weight into adulthood [1,2], impairing both physical and mental health across the life-course [3]. Beyond genetic or epigenetic susceptibilities, unhealthy lifestyle behaviours such as energy-dense, micronutrient-poor dietary intake, and high levels of screen time 
(mainly television viewing) are known to promote overweight and obesity [4,5]. In contrast, a healthier diet and physical activity are known to be protective $[5,6]$.

Traditional approaches to understanding the determinants of adiposity have considered these lifestyle behaviours in isolation; however this approach fails to account for the known correlation or clustering of such behaviours in individuals [7]. Applying more integrative approaches based on pattern analyses, a number of studies conducted in children of preschool-age or older have identified a variety of patterns combining both healthy and unhealthy behaviours [8,9]. The two most commonly observed lifestyle patterns in children are a "Snacking and sedentary" pattern, characterized by high consumption of energy-dense or processed foods along with high screen time (mostly television); and a "Healthy diet and physically active" pattern, often characterized by high consumption of core foods such as fruit and vegetables, along with high levels of physical activity. These two socio-economically differentiated lifestyle patterns are important as they reflect inter-related behavioural combinations that contribute either positively or negatively to energy balance, and have been shown to be associated with higher vs. lower risk of obesity in children [8-12]. Analysing the socio-demographic characteristics of the population groups exposed to co-occurrence or clustering of unhealthy behaviours can also inform the targeting of obesity prevention interventions.

While there is evidence to suggest that dietary habits, physical activity and sedentary screen behaviour are established early and remain constant across childhood [13-15] and into adulthood [16], to our knowledge, only one study (in two-year-olds) has been published on the clustering of lifestyle behaviours prior to preschool age [17]. No study has assessed whether lifestyle patterns appear earlier than two years, nor their persistence throughout early childhood.

To address this gap, the objectives of the present study were to identify lifestyle patterns at 1.5, 3.5 and 5 years, including dietary intake, outdoor time and television viewing time, to assess their associations with maternal education (used as a proxy for socio-economic position), and to investigate the extent to which these patterns are stable from toddlerhood to preschool age.

\section{Materials and Methods}

\subsection{Study Design and Participants}

The Melbourne Infant Feeding Activity and Nutrition Trial (InFANT) and InFANT Extend Programs are separate cluster randomized controlled trials, which have been described in detail elsewhere [18-20]. For both interventions, eligibility involved being a first-time mother of an infant aged around 4 months of age and being able to communicate in English. Of those eligible, $86 \%(n=542)$ and $97 \%(\mathrm{n}=514)$ consented to participate in the InFANT and InFANT Extend Programs, respectively. Given the similarity of studies and measures, we pooled data in order to increase power and therefore robustness of the current analyses.

The InFANT Program was a two arm cluster randomised controlled trial that commenced in 2008. Eligible mothers and their infants were recruited from 62 first-time parent groups organized by the universal Maternal and Child Health service provided across Victoria, Australia [18,19]. Families were randomised at the group level to the control or intervention condition. Parents in the intervention group were offered six 2-h dietitian delivered sessions over 15 months, focusing on parental knowledge, skills, and social support around infant feeding, diet, physical activity, and television viewing. The primary aim was to improve lifestyle behaviours and BMI z-scores. Measures were collected at baseline (mean age 4 months), end of intervention (mean age 20 months), and at follow-ups 2 and 3.5 years post intervention (child age approx. 3.5 and $5 \mathrm{y}$, respectively). Ethical approval was granted by Deakin University's Human Ethics Research Committee (EC175-2007) and the Victorian Office for Children (CDF/07/1138) and the trial was registered with the ISRCTN Register (ISRCTN81847050).

The Extended Infant Feeding, Activity and Nutrition Trial (InFANT Extend) employed similar methodology to the Melbourne InFANT Program [20]. This trial tested the efficacy of an extended (33 vs. 15 month) and enhanced (use of web-based materials, and Facebook ${ }^{\circledR}$ engagement), version of 
the original Melbourne InFANT Program intervention in a new cohort of 514 families from 62 first-time parent groups in Melbourne, Australia, from 2010. Measures were collected at baseline (mean age 4 months), mid-intervention (mean age 19 months), and at intervention conclusion (mean age 38 months). Ethical approval was granted by Deakin (EC-175-2007 (Part 2- 2007-175) and the Department of Education and Early Childhood Development (Victoria, Australia) (2011_001000) and was registered with ACTRN (ACTRN12611000386932).

\subsection{Measurements}

Data used in the current study were collected using self-administered questionnaires provided to the main carer (mostly mothers). Eight variables describing usual dietary intake, outdoor time and television time, available at all relevant time points across both studies, i.e., 1.7, 3.5 and $5 \mathrm{y}$ in InFANT and 1.6 and $3.2 \mathrm{y}$ in InFANT Extend, were included in the pattern analyses. For the sake of simplicity, we refer to these three ages as $1.5 \mathrm{y}, 3.5 \mathrm{y}$ and $5 \mathrm{y}$ throughout the manuscript, although due to scheduling of visits not all children were the exact age at measurement.

\subsubsection{Dietary Intake}

Usual dietary intake over the last month was assessed using a 66-item food frequency questionnaire (FFQ) (under review). Nine possible response categories ranged from 'never or less than once a month' to ' 6 or more times a day'. Portion sizes for each FFQ item were generated based on median portion sizes consumed at each age in the 2007 National Children's Nutrition and Physical Activity Survey data [21] and applied to each FFQ item. Daily consumption of each FFQ food item in grams was calculated by converting the frequency of consumption into daily equivalents (Never $=0 ; 1-3 /$ month $=$ $0.067 ; 1 /$ week $=0.143 ; 2-4 /$ week $=0.429 ; 5-6 /$ week $=0.786 ; 1 /$ day $=1.0 ; 2-3 /$ day $=2.5 ; 4-5 /$ day $=4.5$; $\geq 6 /$ day $=6.0$ times per day) and then multiplying by the calculated median portion size for that food to generate grams per day $(\mathrm{g} / \mathrm{d})$. Consistent with the main dietary outcomes of the InFANT $[18,19]$ and InFANT Extend [20] protocols, six food/drink groups were retained for inclusion in the current lifestyle pattern analysis, i.e., fruit, vegetables, water, sweet drinks, discretionary sweet foods, and discretionary savory foods (all measured in $\mathrm{g} / \mathrm{d}$ ). There was a ceiling effect for water intake, as about half of parents reported their child consumed six or more serves/d (converted to $1050 \mathrm{~g} / \mathrm{d}$ ). This variable was therefore dichotomized as consuming $\geq 1050 \mathrm{~g} / \mathrm{d}$ of water vs. $<1050 \mathrm{~g} / \mathrm{d}$ at each of the time points. Likewise, sweet drinks were consumed by $<50 \%$ of the children at $1.5 \mathrm{y}$ in both InFANT and InFANT Extend, and were dichotomized into consumers vs. non-consumers at this age. Because we used a pattern analysis sensitive to outliers (see Statistical Analyses section), values for outliers were replaced by the maximum values of the remaining distributions at each age in the InFANT and InFANT Extend samples ( $n=17$ and 12 respectively).

\subsubsection{Outdoor Time}

Parents reported the number of minutes (open response) their child spent outside on a typical day. Parent-reported usual outdoor time has been shown to be associated with direct objective measurement of physical activity by accelerometer in preschool-aged children [22]. Values for outliers were replaced by the maximum values of the remaining distributions at each age in InFANT and InFANT Extend samples ( $n=2$ and 3 respectively).

\subsubsection{Television Viewing Time}

Parents reported the number of minutes (open response) their child spent watching or in front of the television on a typical day using items with established reliability [23]. Test-retest reliability in this sample indicated good convergence with intraclass correlation coefficient ICC $=0.69$ at 3 months of age and ICC $>0.79$ for other ages. Values for outliers were replaced by the maximum value of the remaining distributions at each age in InFANT and InFANT Extend ( $n=2$ and 3 respectively). 


\subsubsection{Demographic, Socioeconomic and Anthropometric Factors}

Demographic, socioeconomic and anthropometric data reported at baseline included children's sex and birth weight and mother's country of birth and maternal education level. The latter was defined in three categories: low (secondary school or below), intermediate (trade and certification qualifications) and high (university degree). Maternal education is the indicator most commonly used as a proxy for socio-economic position when considering lifestyle behaviours of children [24]; also, it is consistently and strongly associated with child adiposity in the literature [25] and is less likely to be affected by motherhood, as compared to other indicators such as income and occupation.

\subsubsection{Included Samples}

Of the 542 and 514 mother-child dyads initially recruited to InFANT and InFANT Extend, respectively, 179 and 168 participants dropped out between enrolment and the end of follow-up, i.e., at $5 \mathrm{y}$ in InFANT and at $3.5 \mathrm{y}$ in InFANT Extend ( Figure 1; Figure 2). Additionally, children with missing information on any of the behaviours of interest for the current study were excluded from the analysis ( $n=75$ at $1.5 \mathrm{y} ; n=73$ at $3.5 \mathrm{y}$; and $n=58$ at $5 \mathrm{y}$ for InFANT; $n=109$ at $1.5 \mathrm{y}$; and $n=72$ at $3.5 \mathrm{y}$ for InFANT Extend), resulting in 417, 297 and 305 eligible children for analysis at 1.5, 3.5 and 5 y in InFANT; and 293 and 274 at 1.5 and $3.5 \mathrm{y}$ in InFANT Extend.

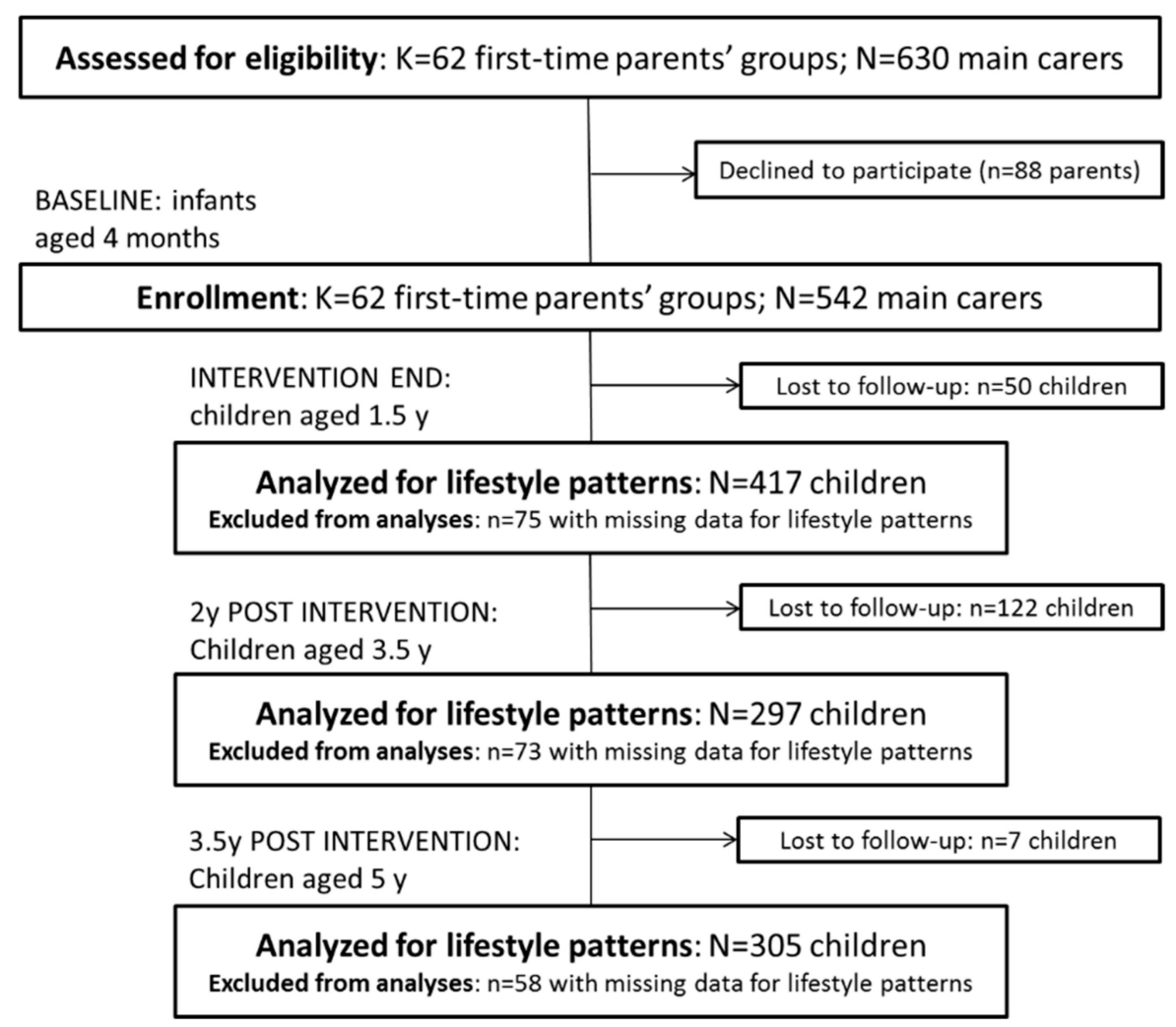

Figure 1. Flow of participants through the Melbourne InFANT Program. 


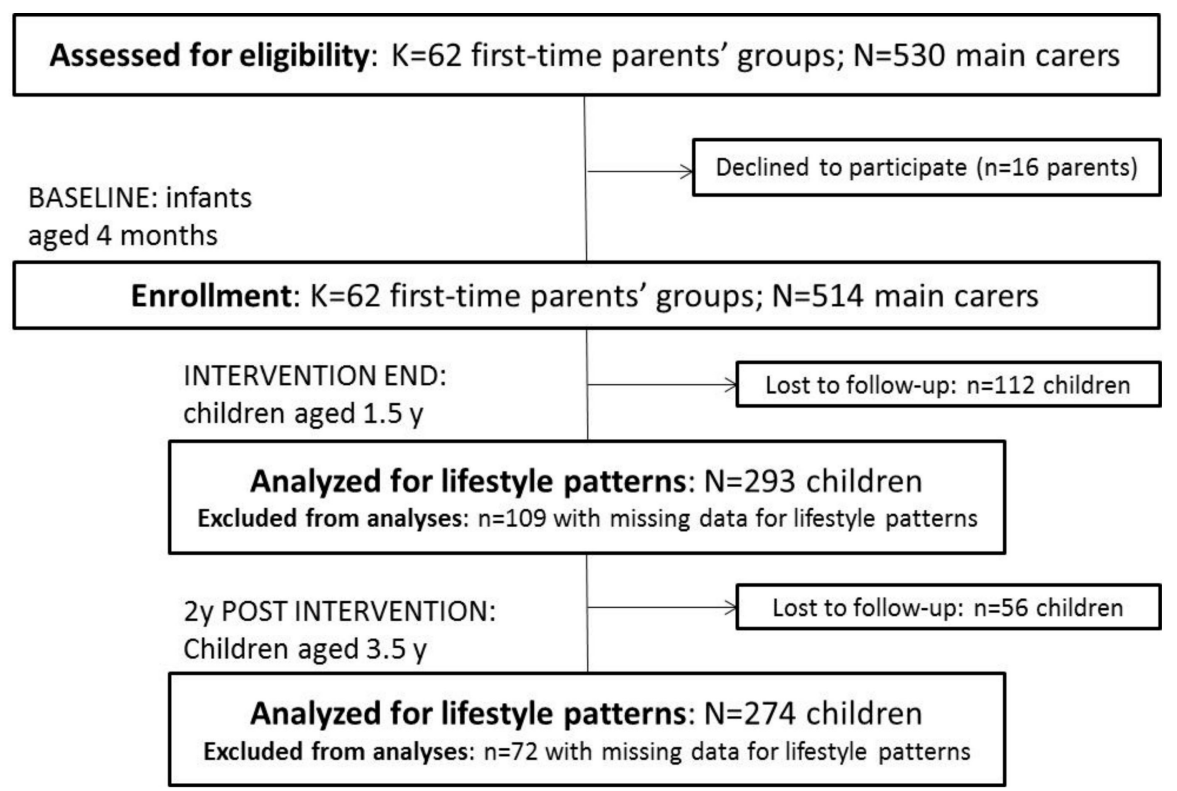

Figure 2. Flow of participants through the Melbourne InFANT Extend Program.

We derived lifestyle patterns for all subjects with complete data at each age. Multi-time-point lifestyle patterns were also assessed in the 247 and 210 children with data available at all three ages in InFANT and at the two ages in InFANT Extend, respectively.

\subsection{Statistical Analyses}

We described the children from the InFANT $(n=417)$ and InFANT Extend $(n=293)$ datasets who had all behavioural data available at approx. $1.5 \mathrm{y}$. Socio-demographic and anthropometric factors were then compared between this sample and the children assessed at baseline (children aged 4 months) but not included in the analysis undertaken at $1.5 \mathrm{y}$, because of loss to follow-up or missing data ( $n=125$ and $n=221$, respectively). Chi-square and Student $t$ tests were used to compare frequencies and means, respectively.

Different data-driven approaches have been used to identify both dietary and lifestyle patterns, in particular principal component analysis (PCA) and cluster analysis (CA). These approaches have shown good reproducibility $[8,9,26]$. PCA allows the synthesis of a large number of supposedly correlated variables into a fewer number of patterns, which are linear combinations of the initial standardized variables: each subject from the analytical sample has a score on each of the patterns derived. CA reduces data into mutually exclusive groups (clusters) based upon individual differences in mean intakes: subjects are member of one given cluster. In the current analysis, we aimed to measure adherence to each of the patterns derived, and not separate children in mutually exclusive groups, which led us to use PCA.

Cross-sectional lifestyle patterns were first derived independently at $1.5 \mathrm{y}$ and $3.5 \mathrm{y}$ (InFANT and InFANT Extend) and $5 \mathrm{y}$ (InFANT) using PCA with varimax rotation of the 8 standardized behavioural variables, i.e., fruit, vegetables, water, sweet drinks, discretionary sweet foods, discretionary savory foods, outdoor and television viewing time. The number of patterns was selected considering eigenvalues $>1.0$, the scree plot and the interpretability of the patterns $[27,28]$. To interpret and label each pattern, we considered the items most strongly related to that pattern, i.e., those for which the absolute value of the loading coefficient (i.e., the correlation of each variable with the given lifestyle pattern) was $>0.25$. The PCA scores for each lifestyle pattern were calculated at the individual level by summing the observed standardized values of each variable, weighted according to the PCA loadings.

Longitudinal, multi-time-point lifestyle patterns were then derived in a separate PCA with varimax rotation, for each study, including simultaneously the standardized variables defined at all 
available time points, i.e., $1.5 \mathrm{y}, 3 \mathrm{y}$ and $5 \mathrm{y}(\operatorname{InFANT}, \mathrm{n}=247)$; and $1.5 \mathrm{y}$ and $3 \mathrm{y}$ (InFANT Extend, $n=210$ ). The same criteria were considered to retain and label the resulting patterns. This longitudinal application of PCA was previously used by Brazionis et al. [29] to assess transition diets in the early years and by Lioret et al. [14] as a longitudinal measure of tracking on dietary patterns. Persistence of patterns from $1.5 \mathrm{y}$ to $5 \mathrm{y}$ ( $\operatorname{InFANT}, n=247$ ) and from $1.5 \mathrm{y}$ to $3.5 \mathrm{y}$ (InFANT Extend, $n=210$ ) was also assessed with Pearson correlation coefficients and associated $p$-values estimated between lifestyle patterns in each dataset. Recommendations for interpreting these correlation coefficients are: low $0.1-0.3$, medium $0.3-0.5$ and high $>0.5$ [30]. Sensitivity pattern analyses were undertaken on the pooled InFANT and InFANT Extend datasets at $1.5 \mathrm{y}(n=710)$ and $3.5 \mathrm{y}(n=571)$. Of note, consistent loading coefficients were observed from PCA independently undertaken in intervention and control groups (results available on request); findings from the whole InFANT and InFANT Extend samples were, however, prioritized to allow a higher statistical power and therefore precision of the estimated scores. Lastly, we performed multivariable linear regression analysis to investigate the relations between a given lifestyle pattern (as the outcome) and maternal education level, adjusting for child sex, age, treatment and using the survey command in Stata (to account for clustering by first-time parent group). This analysis was performed in each of the pooled $1.5 \mathrm{y}$ and $3.5 \mathrm{y}$ samples to avoid multiple comparisons. Adjusted parameter estimates and 95\% CIs were calculated.

\section{Results}

\subsection{Characteristics of the Study Population}

Children were aged on average $1.6(\mathrm{SD}=0.2), 3.6(0.2)$, and $5.0(0.1) \mathrm{y}$ at the three time points in InFANT; and $1.6(0.1)$ and $3.2(0.1) \mathrm{y}$ at the two time points in InFANT Extend. Sample characteristics are presented in Table 1. In InFANT Extend, the 221 children excluded from the analyses at $1.5 \mathrm{y}$ due to loss at follow-up or missing data came from families where the mothers were younger and less likely to have achieved a high education level compared to those retained in the analyses; there were no such differences in InFANT.

\subsection{Characteristics of Lifestyle Patterns}

\section{Cross-Sectional PCA Approach}

Two consistent lifestyle patterns were identified in each of the independent analyses across time points and study populations. Each of these patterns accounted for $18.3-22.0 \%$ of the explained variance (Table 2). Overall, at each time point, one pattern was consistently and positively correlated with intakes of sweet drinks, discretionary sweet foods, discretionary savory foods and television time. We labelled this pattern "Discretionary consumption and TV" (referred to as LP1 in the tables). The other pattern, labelled "Fruit, vegetables and outdoor" (referred to as LP2 in the tables), was mainly characterized by high intake of fruit and vegetables and high levels of outdoor time. When the absolute value of water intake loaded 0.25 , water was either negatively correlated with the "Discretionary consumption and TV" pattern, or positively correlated with the "Fruit, vegetables and outdoor" pattern. Consistent findings were obtained from analyses using the pooled InFANT-InFANT Extend dataset at 1.5 and 3.5 y (Table 3). 
Table 1. Characteristics of the study samples.

\begin{tabular}{|c|c|c|c|c|c|c|}
\hline & $\begin{array}{c}\text { Study Sample } \\
\text { at } 1.5 \mathrm{y}\end{array}$ & $\begin{array}{l}\text { InFANT } \\
\text { Children Included at Baseline but } \\
\text { Excluded from Analyses at } 1.5 \mathrm{y}\end{array}$ & $p$-Value & $\begin{array}{c}\text { Study Sample } \\
\text { at } 1.5 \mathrm{y}\end{array}$ & $\begin{array}{l}\text { InFANT Extend } \\
\text { Children Included at Baseline but } \\
\text { Excluded from Analyses at } 1.5 \mathrm{y}\end{array}$ & $p$-Value \\
\hline$n$ & 417 & 125 & & 293 & 221 & \\
\hline Maternal age at baseline, mean (SD) & $32.4(4.2)$ & $32.1(4.6)$ & 0.54 & $32.5(4.3)$ & $31.4(4.3)$ & 0.008 \\
\hline Maternal education level, $n(\%)$ & & & 0.21 & & & 0.02 \\
\hline Low & $85(20.4)$ & $27(23.9)$ & & $28(9.6)$ & $31(16.6)$ & \\
\hline Intermediate & $98(23.5)$ & $33(29.2)$ & & $83(28.5)$ & $61(32.6)$ & \\
\hline High & $234(56.1)$ & $53(46.9)$ & & $180(61.9)$ & $95(50.8)$ & \\
\hline Mother's country of birth, $n(\%)$ & & & 0.26 & & & 0.10 \\
\hline Australia & $334(80.1)$ & $85(75.2)$ & & $227(78.8)$ & $135(72.2)$ & \\
\hline Other & $83(19.9)$ & $28(24.8)$ & & $61(21.2)$ & $52(27.8)$ & \\
\hline Child sex, $n(\%)$ & & & 0.17 & & & 0.98 \\
\hline Male & $226(54.2)$ & $59(47.2)$ & & $154(52.6)$ & $100(52.4)$ & \\
\hline Female & $191(45.8)$ & $66(52.8)$ & & $139(47.4)$ & $91(47.6)$ & \\
\hline Child birth weight, mean (SD) & $3376.5(595.6)$ & $3361.9(645.1)$ & 0.82 & $3323.2(612.1)$ & $3416.2(554.6)$ & 0.11 \\
\hline
\end{tabular}


Table 2. Behavioural variables distribution and PCA loadings for lifestyle patterns (LP) derived at $1.5 \mathrm{y}, 3.5 \mathrm{y}$ and $5 \mathrm{y}$.

\begin{tabular}{|c|c|c|c|c|c|c|c|c|c|c|c|c|c|c|c|}
\hline \multirow{2}{*}{$\begin{array}{c}\text { Age } \\
\text { Study } \\
n\end{array}$} & \multicolumn{6}{|c|}{$1.5 \mathrm{y}$} & \multicolumn{6}{|c|}{$3.5 \mathrm{y}$} & \multirow{2}{*}{\multicolumn{3}{|c|}{$\begin{array}{c}5 \mathrm{y} \\
\text { InFANT } \\
305\end{array}$}} \\
\hline & \multicolumn{3}{|c|}{$\begin{array}{c}\text { InFANT } \\
417\end{array}$} & \multicolumn{3}{|c|}{$\begin{array}{c}\text { InFANT Extend } \\
293\end{array}$} & \multicolumn{3}{|c|}{$\begin{array}{c}\text { InFANT } \\
297\end{array}$} & \multicolumn{3}{|c|}{$\begin{array}{c}\text { InFANT Extend } \\
274\end{array}$} & & & \\
\hline & $\begin{array}{l}\text { Mean } \\
\text { (SD) }{ }^{a}\end{array}$ & PCA L & adings & $\begin{array}{l}\text { Mean } \\
\text { (SD) }{ }^{a}\end{array}$ & PCA L & adings & $\begin{array}{l}\text { Mean } \\
\text { (SD) }{ }^{a}\end{array}$ & PCA L & adings & $\begin{array}{l}\text { Mean } \\
\text { (SD) }{ }^{a}\end{array}$ & PCA L & adings & $\begin{array}{l}\text { Mean } \\
\text { (SD) }{ }^{a}\end{array}$ & PCA I & adings \\
\hline Behavioural variables & & LP1 ${ }^{b}$ & $\mathrm{LP} 2^{\mathrm{c}}$ & & LP1 b & $\mathrm{LP} 2^{\mathrm{c}}$ & & LP1 b & $\mathrm{LP} 2^{\mathrm{c}}$ & & LP1 b & $\mathrm{LP} 2^{\mathrm{c}}$ & & LP1 ${ }^{b}$ & $\mathrm{LP} 2^{\mathrm{c}}$ \\
\hline Fruit, g/d & $\begin{array}{c}195.3 \\
(113.0)^{\mathrm{d}}\end{array}$ & 0.06 & 0.65 & $\begin{array}{c}270.0 \\
(140.7)\end{array}$ & 0.02 & 0.59 & $\begin{array}{c}248.2 \\
(119.9)\end{array}$ & 0.02 & 0.56 & $\begin{array}{c}275.5 \\
(145.2)\end{array}$ & 0.04 & 0.69 & $\begin{array}{c}258.9 \\
(129.9)\end{array}$ & 0.07 & 0.54 \\
\hline Vegetables, $\mathrm{g} / \mathrm{d}$ & $\begin{array}{c}98.5 \\
(50.4)\end{array}$ & -0.06 & 0.63 & $\begin{array}{l}97.3 \\
(50.7)\end{array}$ & -0.01 & 0.58 & $\begin{array}{c}88.8 \\
(50.3)\end{array}$ & -0.09 & 0.60 & $\begin{array}{l}83.8 \\
(47.1)\end{array}$ & -0.06 & 0.65 & $\begin{array}{c}89.4 \\
(46.8)\end{array}$ & 0.06 & 0.52 \\
\hline Water $\geq 1050 \mathrm{~g} / \mathrm{d}$ & 49.4 & -0.17 & 0.07 & 57.3 & -0.27 & 0.13 & 38.7 & -0.22 & 0.29 & 47.1 & -0.21 & 0.00 & 38.0 & -0.20 & 0.37 \\
\hline Sweet drinks, g/d & 37.6 & 0.50 & 0.07 & 34.8 & 0.53 & 0.02 & $\begin{array}{c}53.9 \\
(79.9)\end{array}$ & 0.44 & -0.05 & $\begin{array}{c}31.4 \\
(44.4)\end{array}$ & 0.50 & 0.07 & $\begin{array}{c}49.9 \\
(71.2)\end{array}$ & 0.47 & -0.09 \\
\hline $\begin{array}{l}\text { Discretionary sweet } \\
\text { foods, g/d }\end{array}$ & $\begin{array}{c}9.6 \\
(10.0)\end{array}$ & 0.54 & -0.07 & $8.3(9.3)$ & 0.58 & 0.00 & $\begin{array}{l}15.0 \\
(11.6)\end{array}$ & 0.48 & 0.00 & $\begin{array}{c}13.3 \\
(10.3)\end{array}$ & 0.47 & 0.03 & $\begin{array}{c}15.2 \\
(12.2)\end{array}$ & 0.57 & 0.12 \\
\hline $\begin{array}{l}\text { Discretionary savory } \\
\text { foods, } \mathrm{g} / \mathrm{d}\end{array}$ & $3.1(4.1)$ & 0.55 & 0.06 & $2.9(3.8)$ & 0.45 & 0.09 & $4.6(5.4)$ & 0.45 & -0.07 & $5.2(5.0)$ & 0.52 & 0.01 & $5.0(5.2)$ & 0.53 & -0.08 \\
\hline $\mathrm{TV}, \min / \mathrm{d}$ & $\begin{array}{c}51.2 \\
(58.0)\end{array}$ & 0.31 & -0.22 & $\begin{array}{c}47.0 \\
(45.6)\end{array}$ & 0.29 & -0.34 & $\begin{array}{l}106.6 \\
(76.8)\end{array}$ & 0.51 & 0.09 & $\begin{array}{c}92.5 \\
(59.1)\end{array}$ & 0.45 & -0.16 & $\begin{array}{c}96.9 \\
(64.4)\end{array}$ & 0.36 & 0.14 \\
\hline Outdoor, min/d & $\begin{array}{l}101.7 \\
(69.9)\end{array}$ & 0.14 & 0.33 & $\begin{array}{l}123.0 \\
(65.4)\end{array}$ & 0.18 & 0.43 & $\begin{array}{l}164.9 \\
(99.2)\end{array}$ & 0.23 & 0.48 & $\begin{array}{c}174.9 \\
(105.8)\end{array}$ & 0.10 & 0.26 & $\begin{array}{l}160.3 \\
(80.2)\end{array}$ & -0.06 & 0.50 \\
\hline$\%$ variance explained & & 18.9 & 18.5 & & 20.8 & 20.1 & & 22.0 & 18.3 & & 21.8 & 18.4 & & 21.0 & 19.4 \\
\hline
\end{tabular}

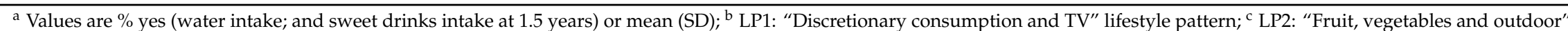
lifestyle pattern. d Fruit was summed consumption of 10 fruits; at $1.5 \mathrm{y}$ in the InFANT sample banana was not reported so fruit was summed consumption of nine fruits. In bold: Factor loadings $<-0.25$ or $>0.25$. 
Table 3. PCA loadings for lifestyle patterns (LP) derived at $1.5 \mathrm{y}$ and $3.5 \mathrm{y}$ in the pooled datasets (InFANT and InFANT Extend studies combined).

\begin{tabular}{|c|c|c|c|c|}
\hline Age & \multicolumn{2}{|c|}{$1.5 \mathrm{y}$} & \multicolumn{2}{|c|}{$3.5 \mathrm{y}$} \\
\hline$n$ & \multicolumn{2}{|c|}{710} & \multicolumn{2}{|c|}{571} \\
\hline $\begin{array}{c}\text { Behavioural } \\
\text { variables }\end{array}$ & LP1 & LP2 & LP1 & LP2 \\
\hline Fruit & 0.03 & 0.62 & 0.04 & 0.67 \\
\hline Vegetables & -0.04 & 0.58 & -0.06 & 0.64 \\
\hline Water & -0.21 & 0.12 & -0.24 & 0.15 \\
\hline Sweet drinks & 0.51 & 0.07 & 0.47 & 0.03 \\
\hline $\begin{array}{l}\text { Discretionary } \\
\text { sweet foods }\end{array}$ & 0.56 & -0.04 & 0.49 & 0.02 \\
\hline $\begin{array}{l}\text { Discretionary } \\
\text { savory foods }\end{array}$ & 0.51 & 0.06 & 0.48 & 0.02 \\
\hline TV & 0.30 & -0.27 & 0.46 & -0.07 \\
\hline Outdoor & 0.15 & 0.43 & 0.15 & 0.33 \\
\hline $\begin{array}{l}\% \text { variance } \\
\text { explained }\end{array}$ & 19.6 & 19.1 & 21.7 & 18.0 \\
\hline Label & $\begin{array}{c}\text { Discretionary } \\
\text { consumption and TV }\end{array}$ & $\begin{array}{l}\text { Fruit, vegetables } \\
\text { and outdoor }\end{array}$ & $\begin{array}{c}\text { Discretionary } \\
\text { consumption and TV }\end{array}$ & $\begin{array}{l}\text { Fruit, vegetables } \\
\text { and outdoor }\end{array}$ \\
\hline
\end{tabular}

\subsection{Persistence of Lifestyle Patterns from Toddlerhood to Preschool Age}

\subsubsection{Longitudinal PCA Approach}

Consistent with the findings in the cross-sectional approach, in longitudinal analyses, we identified the two multi-time-point lifestyle patterns, "Discretionary consumption and TV" and "Fruit, vegetables and outdoor", spanning 1.5 to $5 \mathrm{y}$ in InFANT and 1.5 to $3.5 \mathrm{y}$ in InFANT Extend. These accounted for $13.2-16.4 \%$ of the explained variance (Supplementary Materials Table S1).

\subsubsection{Correlation Approach}

Correlation coefficients between the "Discretionary consumption and TV" pattern observed at $1.5 \mathrm{y}, 3.5 \mathrm{y}$ and $5 \mathrm{y}$ respectively were universally high (0.50-0.63) [30], with corresponding coefficients for the "Fruit, vegetables and outdoor" pattern at different ages being medium to high (0.36-0.60) (Table 4).

Table 4. Pearson correlation coefficients ${ }^{1}$ and $p$-values between lifestyle patterns (LP) scores identified at $1.5 \mathrm{y}, 3.5 \mathrm{y}$ and $5 \mathrm{y}$.

\begin{tabular}{ccccccc}
\hline \multirow{2}{*}{ Study $(n)$} & \multirow{2}{*}{ Age } & & \multicolumn{2}{c}{$3.5 \mathrm{y}$} & \multicolumn{2}{c}{$5 \mathrm{y}$} \\
\cline { 4 - 7 } & & & LP1 & LP2 & LP1 & LP2 \\
\hline \multirow{2}{*}{ InFANT (247) } & \multirow{2}{*}{$1.5 \mathrm{y}$} & LP1 & $0.52^{* * *}$ & $-0.20^{* *}$ & $0.50^{* * *}$ & $-0.17^{* * *}$ \\
& & LP2 & -0.05 & $0.47^{* * *}$ & -0.12 & $0.36^{* * *}$ \\
& \multirow{2}{*}{$3.5 \mathrm{y}$} & LP1 & & & $0.63^{* * *}$ & $-0.13^{*}$ \\
& & LP2 & & & $-0.23^{* * *}$ & $0.60^{* * *}$ \\
InFANT Extend $(210)$ & \multirow{2}{*}{$1.5 \mathrm{y}$} & LP1 & $0.51^{* * *}$ & -0.06 & & \\
& & LP2 & -0.04 & $0.51^{* * *}$ & & \\
\end{tabular}

${ }^{*} p<0.05, \overline{* *} p<0.01,{ }^{* * *} p<0.001, n=989 ;{ }^{1}$ Recommendations for interpreting these correlation coefficients: low $<0.3$, moderate 0.3-0.6 and high >0.6 [30]; LP1: "Discretionary consumption and TV" lifestyle pattern; LP2: "Fruit, vegetables and outdoor" lifestyle pattern. 


\subsection{Associations with Maternal Education}

The "Discretionary consumption and TV" pattern was inversely associated with maternal education level at both 1.5 (significant trend) and $3.5 \mathrm{y}$ in the pooled datasets (Table 5). Conversely, the "Fruit, vegetables and outdoor" pattern was positively associated with maternal education at $1.5 \mathrm{y}$.

Table 5. Lifestyle pattern scores in the pooled InFANT and InFANT Extend datasets according to maternal education level (Adjusted ${ }^{a} \beta$ parameter estimates and 95\% confidence intervals).

\begin{tabular}{|c|c|c|c|c|c|c|c|c|}
\hline \multirow{3}{*}{$\begin{array}{c}\text { Lifestyle Pattern } \\
\text { Age }\end{array}$} & \multicolumn{4}{|c|}{ LP1 } & \multicolumn{4}{|c|}{ LP2 } \\
\hline & \multicolumn{2}{|c|}{$1.5 \mathrm{y}$} & \multicolumn{2}{|c|}{$3.5 \mathrm{y}$} & \multicolumn{2}{|c|}{$1.5 \mathrm{y}$} & \multicolumn{2}{|c|}{$3.5 \mathrm{y}$} \\
\hline & $\beta$ & $95 \% \mathrm{CI}$ & $\beta$ & $95 \% \mathrm{CI}$ & $\beta$ & $95 \% \mathrm{CI}$ & $\beta$ & $95 \% \mathrm{CI}$ \\
\hline$n$ & 708 & & 567 & & 708 & & 567 & \\
\hline \multicolumn{9}{|l|}{$\begin{array}{l}\text { Maternal } \\
\text { education }\end{array}$} \\
\hline Low & Ref & & Ref & & Ref & & Ref & \\
\hline Intermediate & 0.01 & $\begin{array}{c}-0.29 \\
0.31\end{array}$ & -0.34 & $\begin{array}{c}-0.77 \\
0.08\end{array}$ & 0.18 & $\begin{array}{c}-0.12 \\
0.49\end{array}$ & -0.15 & $\begin{array}{c}-0.51 \\
0.20\end{array}$ \\
\hline High & -0.22 & $\begin{array}{c}-0.50 \\
0.05\end{array}$ & -0.65 & $\begin{array}{l}-0.98 \\
-0.32\end{array}$ & 0.34 & $\begin{array}{l}0.08 \\
0.59\end{array}$ & -0.01 & $\begin{array}{c}-0.33 \\
0.32\end{array}$ \\
\hline$p$ value & 0.06 & & 0.0002 & & 0.03 & & 0.45 & \\
\hline$p$ trend & 0.037 & & 0.000 & & 0.009 & & & \\
\hline
\end{tabular}

a Multivariable linear regression analyses accounted for child sex, age, treatment and clustering by first-time parent group. LP1: "Discretionary consumption and TV" lifestyle pattern; LP2: "Fruit, vegetables and outdoor" lifestyle pattern.

\section{Discussion}

To the best of our knowledge, this study is the first to show that lifestyle patterns, namely "Discretionary consumption and TV" and "Fruit, vegetables and outdoor", are established by 1.5 years of age. Further, there is evidence of persistence of these lifestyle patterns across early childhood, and that these patterns are influenced by maternal education.

Similar patterns to those identified in this study have been reported in other studies of early childhood populations. In the study most comparable to ours, using PCA, Gubbels et al. [17] found a "Snacking-sedentary" pattern in toddlers aged $2 \mathrm{y}$ in the Netherlands, which was characterized by eating snacks, drinking sugar-sweetened beverages, television viewing and computer use. Lifestyle patterns characterized by less healthy foods and drinks, and screen time, have also been described using either PCA or cluster analysis in children aged 3-6 years across Europe [31-33]. This consistency across countries, datasets and methods used to derive such patterns has also been confirmed in school-aged children [8-10,34-36]. Consistent with our findings, these lifestyle patterns were typically inversely associated with parental socio-economic positions in other studies, most commonly approximated by maternal education $[8,9,35]$. The co-occurrence between television viewing and unhealthy dietary habits has often been described in the literature. Proposed mechanisms include: watching TV or movies as a hand-free passive occupation enables snacking; exposure to advertising of junk food and fast food when watching TV may increase children's requests for those particular foods and products; and viewing screens while eating distracts from internal satiety cues potentially leading to overconsumption [37-39]. Notably, a lifestyle pattern characterized by consumption of discretionary foods and drinks, and elevated screen time, is the pattern most often associated with child overweight in the literature $[8-12,35,36]$.

The lifestyle pattern characterized by the high consumption of core foods such as fruit and vegetables, along with increased time spent in physical activities (referred to here as "Fruit, vegetables and outdoor") has been less frequently identified in other studies, and only in children aged $3 \mathrm{y}$ or older [8-10,36]. Among pre-schoolers, Lioret et al. [31] identified a "Varied food and physically active" pattern in 3-6 y French children; and Miguel-Berges et al. [33] identified a "Healthy lifestyle" pattern 
in European preschool-aged children (3.5-5.5 y), characterized not only by high water, fruit, vegetable intake and sports participation, but also by increased sleep time; and reduced soft drinks and screen time. A very similar healthy pattern was described in Australian children aged 6-7 y [10]. This lifestyle pattern, combining a range of healthy behaviours, has most often been found to be positively associated with parental socio-economic position (often approximated by maternal education) [8-10], which our findings confirm at $1.5 \mathrm{y}$. This socioeconomic disparity may reflect varying family knowledge, social norms, attitudes, and lifestyles, within an environment providing differential access to both healthy food and safe places to play outside. It is important to note that young children are mostly controlled and influenced by their parents, who are also usually their main role models and providers [40]. There is some evidence to suggest that children's lifestyle, whether diet, physical activity or sedentary behaviours, is strongly associated with their parents' diet and physical activity, and with parent capacity to control the availability of healthy foods at home and engage their children in physical activities [41-43]. Potentially, the same parents who are aware of, value, and have the resources to provide a healthy diet, will also be aware of, value and have the resources to promote physical activity and limit screen time, leading to an "overall healthy" lifestyle pattern.

Tracking from early life of each of the lifestyle behaviours under study has been previously described [13-15]. The present study, however, further highlights that combinations of either healthy or unhealthy behaviours persist from as early as $1.5 \mathrm{y}$, which provides new and important insights into the genesis of these behaviours. Not only have we shown that similar lifestyle patterns were overall highly correlated between 1.5, 3.5 and $5 \mathrm{y}$ in InFANT, especially for unhealthy behaviours, but we have also derived two longitudinal multi-time point patterns, each characterized by repeated exposures to the same behaviours over time. Higher scores for "Discretionary consumption and TV at 1.5, 3.5 and $5 y^{\prime \prime}$ reflect higher tracking for this particular pattern of behaviours, whereas higher scores for the "Fruit, vegetables and outdoor at 1.5, 3.5 and $5 \mathrm{y}$ " pattern may be interpreted as a stable adherence to a healthy lifestyle across early childhood. Consistent findings and interpretations from both InFANT and InFANT Extend datasets suggests robustness of these patterns across samples. Another study examining the clustering of energy balance-related behaviours in a smaller sample of Australian school-aged children (123 aged 5-6 and 87 children aged 10-12 y at baseline) found fair to moderate persistence of lifestyle cluster membership over three years, providing further evidence for the early establishment of clusters of obesogenic lifestyle behaviours [35]. In that study, the clusters of obesity-promoting behaviours were hypothesized to lead to cumulative increases in positive energy balance over the life course.

These findings have important implications for conceptualizing early obesity prevention policy and practice. A better understanding of when, which and in whom behaviours co-occur and start to track collectively over time informs the design and delivery of interventions targeting groups of behaviours rather than individual behaviours. Importantly, these findings will also inform policy that guides provision of early childhood care, ensuring that the large numbers of children in childcare are supported to achieve healthy behavioural patterns. It has also been hypothesized that with behaviours that co-occur, change in one may support change in the other, highlighting the potential for multiple-behaviour interventions to have a greater impact on public health than single-behaviour interventions, especially for preventing multifactorial health conditions [44,45]. Interventions focusing on reducing television time, along with the promotion of physical activity engagement and healthy eating, therefore may be promising, especially in children of less educated mothers. Such integrated interventions may have the potential to provide a synergistic impact on health behaviours that goes beyond the simple additive effects of focusing on individual behaviours consecutively. This may help explain the greater success of multi-component interventions in the prevention of childhood overweight $[44,45]$. 


\section{Limitations and Strengths}

In interpreting the findings of the present study, it is important to acknowledge several limitations. Parental self-report of children's behaviours is subject to measurement error, i.e., social desirability bias and relative imprecision as compared to objective measurements (e.g., using accelerometry for physical activity). With respect to food intake, frequency of consumption has been shown to be the major determinant of dietary intake [46]. The FFQ used across the studies was developed specifically for this age group and has been validated against three $24 \mathrm{~h}$ recalls in children aged 1.5, 3.5 and $5.0 \mathrm{y}$ : fair agreement for most nutrient and food intakes was observed, with acceptable rankings of intake suitable for use in PCA (which is based on the underlying correlation matrix) (under review). Additionally, outdoor time has been positively associated with objectively-measured PA in pre-schoolers [22], and both outdoor and television viewing time have been found to be prospectively related to adiposity in early childhood [6,47]. Of note, whereas accelerometry does provide objective information on duration and intensity of activities, the measurements of physical activity and sedentary screen behaviours used in the present study provide information on the context in which they take place (i.e., outdoor and television viewing), which is potentially useful for designing prevention interventions. The present study involved PCA, which is an exploratory statistical alternative among pattern analyses. Strict comparison with other findings is not straightforward due to the data driven approach to pattern analysis, with different methodologies employed in the collection of behavioural data, subjective redistribution of the former items (e.g., foods) into the variables used (e.g., food groups), the transformation of these variables (e.g., standardisation), the methods of rotation of components (if any) employed, and the number of patterns identified-all complicating the opportunities for comparison. Despite these potential variations in methodology, various reviews $[8,9,26]$ have reported fairly good reproducibility between most studies which have identified either dietary or lifestyle patterns. This consistency and therefore robustness was confirmed in the present study. Regarding representativeness, although all levels of maternal education were represented in this study, the sample is generally well educated, with a majority having tertiary education. In addition, mothers excluded from the analyses tended to be less educated and younger, which is a common trait of cohort studies. Whereas these characteristics may have implications for generalizability, the lifestyle patterns derived here are rather consistent with other studies, both cross-sectional and longitudinal, performed in preschool and school-aged children in Australia and overseas.

Particular strengths of the study include consistent measures for a variety of behaviours across studies and time-points, relatively large sample sizes (particularly for pooled analyses), the longitudinal design, and the integrative approach of health behaviours.

\section{Conclusion}

In conclusion, healthy ("Fruit, vegetables and outdoor") and unhealthy ("Discretionary consumption and TV") lifestyle patterns were identified in the present study. These patterns were evident as early as $1.5 \mathrm{y}$ of age, and were extremely robust-across samples and at different child ages. Similar patterns have been observed in a number of other studies carried out in older children [7-9,29-32]. Such early clustering and tracking of healthy and unhealthy energy balance-related behaviours suggest there may be shared antecedents common to the individual behaviours that could be targeted for intervention. Finally, the association with maternal education level indicates that such multiple behaviour interventions may be more effective to prevent child overweight if adapted to the level of family social disadvantage.

Supplementary Materials: The following are available online at http://www.mdpi.com/2072-6643/12/3/724/s1. Table S1: PCA loadings for the multi-time-point lifestyle patterns (LP) in InFANT from $1.5 \mathrm{y}$ to $5 \mathrm{y}$ and in InFANT Extend from $1.5 \mathrm{y}$ to $3.5 \mathrm{y}$.

Author Contributions: S.L. conceptualized the study, conducted the analyses, and drafted the initial manuscript; K.J.C. and K.D.H. conceptualized and led the Infant and Infant Extend Studies; K.J.C. and K.D.H. contributed to the conceptualization of the current study and the analytic approach, contributed to the interpretation of 
results, drafted and edited the manuscript; G.A. guided the statistical analysis, conducted some of the analyses, contributed to the interpretation of results, drafted and edited the manuscript; K.J.C. and S.A.M. conducted the dietary data collection; S.A.M., A.J.C., K.J.C., K.D.H. and J.S. contributed to the interpretation of results, drafted and edited the manuscript. All authors approved the final manuscript as submitted and agree to be accountable for all aspects of the work. All authors have read and agreed to the published version of the manuscript.

Funding: The Melbourne InFANT Program follow-ups were funded by a National Health and Medical Research Council Project Grant (APP1008879); the InFANT Extend Program was funded by a World Cancer Research Fund grant (no. 2010/244). SL was supported by an Alfred Deakin Postdoctoral fellowship (Deakin University, 2010-2012), a sabbatical at IPAN in 2018 funded by INSERM and The French National Research Agency grant (ANR-19-CE36-0006). AJC is the recipient of an Australian Research Council Discovery Early Career Researcher Awards (project number DE160100141). SAM is supported by an NHMRC Career Development Fellowship Level 2 (ID1104636). JS was supported by a National Health and Medical Research Council Principal Research Fellowship during this study (APP1026216). KDH is supported by an Australian Research Council Future Fellowship (FT130100637) and an Honorary Heart Foundation of Australia Future Leader Fellowship (100370).

Conflicts of Interest: The authors declare no conflict of interest. The funders had no role in the design of the study; in the collection, analyses, or interpretation of data; in the writing of the manuscript, or in the decision to publish the results.

\section{References}

1. Simmonds, M.; Llewellyn, A.; Owen, C.G.; Woolacott, N. Predicting adult obesity from childhood obesity: A systematic review and meta-analysis. Obes. Rev. 2016, 17, 95-107. [CrossRef] [PubMed]

2. Kim, J.; Lee, I.; Lim, S. Overweight or obesity in children aged 0 to 6 and the risk of adult metabolic syndrome: A systematic review and meta-analysis. J. Clin. Nurs. 2017, 26, 3869-3880. [CrossRef] [PubMed]

3. Reilly, J.J.; Kelly, J. Long-term impact of overweight and obesity in childhood and adolescence on morbidity and premature mortality in adulthood: Systematic review. Int. J. Obes. (Lond) 2011, 35, 891-898. [CrossRef] [PubMed]

4. World Health Organization (WHO); Food Agriculture Organization (FAO). Diet, Nutrition, and The Prevention of Chronic Diseases; Report of a joint WHO/FAO Expert Conultation; WHO: Geneva, Switzerland, 2003.

5. Swinburn, B.A.; Caterson, I.; Seidell, J.C.; James, W.P. Diet, nutrition and the prevention of excess weight gain and obesity. Public Health Nutr. 2004, 7, 123-146. [PubMed]

6. te Velde, S.J.; van Nassau, F.; Uijtdewilligen, L.; van Stralen, M.M.; Cardon, G.; De Craemer, M.; Manios, Y.; Brug, J.; Chinapaw, M.J. Energy balance-related behaviours associated with overweight and obesity in preschool children: A systematic review of prospective studies. Obes Rev. 2012, 13 (Suppl. 1), 56-74. [CrossRef]

7. McAloney, K.; Graham, H.; Law, C.; Platt, L. A scoping review of statistical approaches to the analysis of multiple health-related behaviours. Prev. Med. 2013, 56, 365-371. [CrossRef]

8. Gubbels, J.S.; van Assema, P.; Kremers, S.P. Physical Activity, Sedentary Behavior, and Dietary Patterns among Children. Curr. Nutr. Rep. 2013, 2, 105-112. [CrossRef]

9. Leech, R.M.; McNaughton, S.A.; Timperio, A. The clustering of diet, physical activity and sedentary behavior in children and adolescents: A review. Int. J. Behav. Nutr. Phys. Act. 2014, 11, 4. [CrossRef]

10. Magee, C.A.; Caputi, P.; Iverson, D.C. Patterns of health behaviours predict obesity in Australian children. J. Paediatr. Child Health 2013, 49, 291-296. [CrossRef]

11. Santaliestra-Pasias, A.M.; Mouratidou, T.; Reisch, L.; Pigeot, I.; Ahrens, W.; Marild, S.; Molnar, D.; Siani, A.; Sieri, S.; Veidebaum, T.; et al. Clustering of lifestyle behaviours and relation to body composition in European children. The IDEFICS study. Eur. J. Clin. Nutr. 2015, 69, 811-816. [CrossRef]

12. Wadolowska, L.; Hamulka, J.; Kowalkowska, J.; Kostecka, M.; Wadolowska, K.; Biezanowska-Kopec, R.; Czarniecka-Skubina, E.; Kozirok, W.; Piotrowska, A. Prudent-Active and Fast-Food-Sedentary DietaryLifestyle Patterns: The Association with Adiposity, Nutrition Knowledge and Sociodemographic Factors in Polish Teenagers-The ABC of Healthy Eating Project. Nutrients 2018, 10, 1988. [CrossRef]

13. Jones, R.A.; Hinkley, T.; Okely, A.D.; Salmon, J. Tracking physical activity and sedentary behavior in childhood: A systematic review. Am. J. Prev. Med. 2013, 44, 651-658. [CrossRef]

14. Lioret, S.; Betoko, A.; Forhan, A.; Charles, M.A.; Heude, B.; de Lauzon-Guillain, B. Dietary patterns track from infancy to preschool age: Cross-sectional and longitudinal perspectives. J. Nutr. 2015, 145, 775-782. 
15. Luque, V.; Escribano, J.; Closa-Monasterolo, R.; Zaragoza-Jordana, M.; Ferre, N.; Grote, V.; Koletzko, B.; Totzauer, M.; Verduci, E.; ReDionigi, A.; et al. Unhealthy Dietary Patterns Established in Infancy Track to Mid-Childhood: The EU Childhood Obesity Project. J. Nutr. 2018, 148, 752-759. [CrossRef]

16. Mikkila, V.; Rasanen, L.; Raitakari, O.T.; Pietinen, P.; Viikari, J. Consistent dietary patterns identified from childhood to adulthood: The cardiovascular risk in Young Finns Study. Br. J. Nutr. 2005, 93, 923-931. [CrossRef]

17. Gubbels, J.S.; Kremers, S.P.; Stafleu, A.; Dagnelie, P.C.; de Vries, S.I.; de Vries, N.K.; Thijs, C. Clustering of dietary intake and sedentary behavior in 2-year-old children. J. Pediatr. 2009, 155, 194-198. [CrossRef]

18. Campbell, K.; Hesketh, K.; Crawford, D.; Salmon, J.; Ball, K.; McCallum, Z. The Infant Feeding Activity and Nutrition Trial (INFANT) an early intervention to prevent childhood obesity: Cluster-randomised controlled trial. BMC Public Health 2008, 8, 103. [CrossRef] [PubMed]

19. Hesketh, K.D.; Campbell, K.; Salmon, J.; McNaughton, S.A.; McCallum, Z.; Cameron, A.; Ball, K.; Gold, L.; Andrianopoulos, N.; Crawford, D. The Melbourne Infant Feeding, Activity and Nutrition Trial (InFANT) Program follow-up. Contemp. Clin. Trials. 2013, 34, 145-151. [CrossRef]

20. Campbell, K.J.; Hesketh, K.D.; McNaughton, S.A.; Ball, K.; McCallum, Z.; Lynch, J.; Crawford, D.A. The extended Infant Feeding, Activity and Nutrition Trial (InFANT Extend) Program: A cluster-randomized controlled trial of an early intervention to prevent childhood obesity. BMC Public Health. 2016, 16, 166. [CrossRef] [PubMed]

21. Australian Food Supplement and Nutrient Database (AUSNUT 2007) [Internet] FSANZ 2007. Available online: http://www.foodstandards.gov.au/science/monitoringnutrients/ausnut/Pages/ausnut2007.aspx (accessed on 27 January 2020).

22. Burdette, H.L.; Whitaker, R.C.; Daniels, S.R. Parental report of outdoor playtime as a measure of physical activity in preschool-aged children. Arch. Pediatr. Adolesc. Med. 2004, 158, 353-357. [CrossRef] [PubMed]

23. Hinkley, T.; Salmon, J.; Okely, A.D.; Crawford, D.; Hesketh, K. The HAPPY study: Development and reliability of a parent survey to assess correlates of preschool children's physical activity. J. Sci. Med. Sport 2012, 15, 407-417. [CrossRef] [PubMed]

24. Ensminger, M.; Fothergill, K. A decade of measuring SES: What it tells us and where to go from here. In Socioeconomic Status, Parenting, and Child Development; Bornstein, M., Bradley, R., Eds.; Lawrence Erlbaum Associates: Mahwah, NJ, USA, 2003; pp. 13-28.

25. Shrewsbury, V.; Wardle, J. Socioeconomic Status and Adiposity in Childhood: A Systematic Review of Cross-sectional Studies 1990-2005. Obesity (Silver Spring) 2008, 16, 275-284. [CrossRef]

26. Newby, P.K.; Tucker, K.L. Empirically derived eating patterns using factor or cluster analysis: A review. Nutr. Rev. 2004, 62, 177-203. [CrossRef] [PubMed]

27. Cattell, R.B. The scree test for the number of factors. Multivar. Behav. Res. 1966, 1, 245-276. [CrossRef] [PubMed]

28. Kline, P. An Easy Guide to Factor Analysis; Routledge: London, UK, 1994.

29. Brazionis, L.; Golley, R.K.; Mittinty, M.N.; Smithers, L.G.; Emmett, P.; Northstone, K.; Lynch, J.W. Characterization of transition diets spanning infancy and toddlerhood: A novel, multiple-time-point application of principal components analysis. Am. J. Clin. Nutr. 2012, 95, 1200-1208. [CrossRef]

30. Cohen, J. Statistical Power Analysis for the Behavioural Science; Hillsdale Erlbaum: New York, NY, USA, 1988.

31. Lioret, S.; Touvier, M.; Lafay, L.; Volatier, J.L.; Maire, B. Dietary and physical activity patterns in French children are related to overweight and socioeconomic status. J. Nutr. 2008, 138, 101-107. [CrossRef]

32. Gubbels, J.S.; Kremers, S.P.; Goldbohm, R.A.; Stafleu, A.; Thijs, C. Energy balance-related behavioural patterns in 5-year-old children and the longitudinal association with weight status development in early childhood. Public Health Nutr. 2012, 9, 1-9. [CrossRef]

33. Miguel-Berges, M.L.; Zachari, K.; Santaliestra-Pasias, A.M.; Mouratidou, T.; Androutsos, O.; Iotova, V.; Galcheva, S.; De Craemer, M.; Cardon, G.; Kulaga, Z.; et al. Clustering of energy balance-related behaviours and parental education in European preschool children: The ToyBox study. Br. J. Nutr. 2017, 118, 1089-1096. [CrossRef]

34. Leech, R.M.; McNaughton, S.A.; Timperio, A. Clustering of children's obesity-related behaviours: Associations with sociodemographic indicators. Eur. J. Clin. Nutr. 2014, 68, 623-628. [CrossRef] 
35. Leech, R.M.; McNaughton, S.A.; Timperio, A. Clustering of diet, physical activity and sedentary behaviour among Australian children: Cross-sectional and longitudinal associations with overweight and obesity. Int. J. Obes. (Lond) 2015, 39, 1079-1085. [CrossRef]

36. Bel-Serrat, S.; Ojeda-Rodriguez, A.; Heinen, M.M.; Buoncristiano, M.; Abdrakhmanova, S.; Duleva, V.; Sant'Angelo, V.F.; Fijalkowska, A.; Hejgaard, T.; Huidumac, C.; et al. Clustering of Multiple Energy Balance-Related Behaviors in School Children and its Association with Overweight and Obesity-WHO European Childhood Obesity Surveillance Initiative (COSI 2015(-)2017). Nutrients 2019, 11, 511. [CrossRef] [PubMed]

37. Temple, J.L.; Giacomelli, A.M.; Kent, K.M.; Roemmich, J.N.; Epstein, L.H. Television watching increases motivated responding for food and energy intake in children. Am. J. Clin. Nutr. 2007, 85, 355-361. [CrossRef] [PubMed]

38. Rey-Lopez, J.P.; Vicente-Rodriguez, G.; Biosca, M.; Moreno, L.A. Sedentary behaviour and obesity development in children and adolescents. Nutr. Metab. Cardiovasc. Dis. 2008, 18, 242-251. [CrossRef] [PubMed]

39. Dalton, M.A.; Longacre, M.R.; Drake, K.M.; Cleveland, L.P.; Harris, J.L.; Hendricks, K.; Titus, L.J. Child-targeted fast-food television advertising exposure is linked with fast-food intake among pre-school children. Public Health Nutr. 2017, 20, 1548-1556. [CrossRef] [PubMed]

40. Golan, M.; Crow, S. Parents are key players in the prevention and treatment of weight-related problems. Nutr. Rev. 2004, 62, 39-50. [CrossRef]

41. Cleland, V.; Timperio, A.; Salmon, J.; Hume, C.; Baur, L.A.; Crawford, D. Predictors of time spent outdoors among children: 5-year longitudinal findings. J. Epidemiol. Community Health 2010, 64, 400-406. [CrossRef]

42. Rodenburg, G.; Oenema, A.; Pasma, M.; Kremers, S.P.; van de Mheen, D. Clustering of food and activity preferences in primary school children. Appetite 2013, 60, 123-132. [CrossRef]

43. Lioret, S.; Cameron, A.J.; McNaughton, S.A.; Crawford, D.; Spence, A.C.; Hesketh, K.; Campbell, K.J. Association between maternal education and diet of children at 9 months is partially explained by mothers' diet. Matern. Child Nutr. 2015, 11, 936-947. [CrossRef]

44. Prochaska, J.J.; Spring, B.; Nigg, C.R. Multiple health behavior change research: An introduction and overview. Prev. Med. 2008, 46, 181-188. [CrossRef]

45. Prochaska, J.O. Multiple Health Behavior Research represents the future of preventive medicine. Prev. Med. 2008, 46, 281-285. [CrossRef]

46. Noethlings, U.; Hoffmann, K.; Bergmann, M.M.; Boeing, H. Portion size adds limited information on variance in food intake of participants in the EPIC-Potsdam study. J. Nutr. 2003, 133, 510-515. [CrossRef] [PubMed]

47. Saldanha-Gomes, C.; Heude, B.; Charles, M.A.; de Lauzon-Guillain, B.; Botton, J.; Carles, S.; Forhan, A.; Dargent-Molina, P.; Lioret, S. Prospective associations between energy balance-related behaviors at 2 years of age and subsequent adiposity: The EDEN mother-child cohort. Int. J. Obes. (Lond) 2017, 41, 38-45. [CrossRef] [PubMed]

(C) 2020 by the authors. Licensee MDPI, Basel, Switzerland. This article is an open access article distributed under the terms and conditions of the Creative Commons Attribution (CC BY) license (http://creativecommons.org/licenses/by/4.0/). 